\title{
BMJ Open Postdischarge service utilisation and outcomes among Chinese and South Asian psychiatric inpatients in Ontario, Canada: a population-based cohort study
}

Maria Chiu, ${ }^{1,2}$ Evgenia Gatov, ${ }^{1}$ Juveria Zaheer, ${ }^{3,4}$ Michael Lebenbaum, ${ }^{1}$ Longdi Fu, ${ }^{1}$ Alice Newman, ${ }^{1}$ Paul Kurdyak ${ }^{1,3,4}$

To cite: Chiu M, Gatov E, Zaheer J, et al. Postdischarge service utilisation and outcomes among Chinese and South Asian psychiatric inpatients in Ontario, Canada: a populationbased cohort study. BMJ Open 2018;8:e020156. doi:10.1136/ bmjopen-2017-020156

- Prepublication history and additional material for this paper are available online. To view these files, please visit the journal online (http://dx.doi. org/10.1136/bmjopen-2017020156).

Received 17 October 2017 Revised 23 November 2017 Accepted 5 December 2017

\section{CrossMark}

${ }^{1}$ Mental Health and Addictions Program, Institute for Clinical Evaluative Sciences, Toronto, Canada

${ }^{2}$ University of Toronto, Institute for Health Policy Management and Evaluation, Toronto, Canada ${ }^{3}$ Institute for Mental Health Policy Research, Toronto, Canada

${ }^{4}$ Department of Psychiatry, Faculty of Medicine, University of Toronto, Toronto, Canada

Correspondence to

Dr Maria Chiu;

maria.chiu@ices.on.ca

\section{ABSTRACT}

Objective We sought to examine the short-term and long-term impacts of psychiatric hospitalisations among patients of Chinese and South Asian origin.

Design Retrospective population-based cohort study using linked health administrative data.

Setting We examined all adult psychiatric inpatients discharged between 1 April 2006 and 31 March 2014 in Ontario, Canada, who were classified as Chinese, South Asian and all other ethnicities (ie, 'general population') using a validated algorithm. We identified 2552 Chinese, 2439 South Asian and 127142 general population patients. Primary and secondary outcome measures We examined psychiatric severity measures at admission and discharge and performed multivariable logistic regression analyses to examine 30-day, 180-day and 365-day postdischarge service utilisation and outcomes, comparing each of the ethnic groups with the reference population, after adjustment for age, sex, income, education, marital status, immigration status, community size and discharge diagnosis.

Results Despite presenting to hospital with greater illness severity, Asian psychiatric inpatients had shorter lengths of hospital stay and greater absolute improvements in mental health and functional status at discharge compared with other inpatients. After hospitalisation, Chinese patients were more likely to visit psychiatrists and South Asian patients were more likely to seek mental healthcare from general practitioners. They were also less likely to have a psychiatric readmission or die 1 year following hospitalisation (adjusted $\mathrm{OR}_{\text {chinese }}=0.87 ; 95 \% \mathrm{Cl} 0.79$ to 0.97 ; adjusted $\mathrm{OR}_{\text {south Asian }}=0.82,95 \% \mathrm{Cl} 0.73$ to 0.91 ). Findings were consistent across genders, psychiatric diagnoses and immigrant groups.

Conclusion Once hospitalised, patients of Chinese and South Asian origin fared as well as or better than general population patients at discharge and following discharge, and had a positive trajectory of psychiatric service utilisation.

\section{INTRODUCTION}

Improving discharge and postdischarge outcomes following a psychiatric hospitalisation and reducing costly readmissions are
Strengths and limitations of this study

- This large population-based study used health administrative databases in a Canadian single-payer healthcare system, which has a high proportion of immigrants and one of the largest Asian populations outside of Asia, making it an ideal setting to study cross-cultural differences.

- Psychiatric inpatients of Chinese and South Asian origin were examined separately, unlike other studies that combined these and other ethnic groups.

- Regression adjustment for a multitude of diagnostic and sociodemographic covariates, and stratification by sex, diagnostic and immigration status allowed for a thorough assessment of the independent effect of ethnicity.

- Low sensitivity of the surnames algorithm used for ethnicity ascertainment may have resulted in misclassification.

- The reference group was heterogeneous and encompassed all other ethnicities, including black African and Caribbean inpatients.

major objectives for mental health systems that offer community care. ${ }^{1}$ The transition from inpatient to outpatient treatment is a critical time, as many patients disengage from care. ${ }^{2}$ Although there is growing interest in the role of ethnicity in the development of mental illness, there has been minimal investigation into its influence on inpatient and postdischarge outcomes. Most previous studies have focused on black African or Caribbean inpatients, demonstrating increased rates of early termination of treatment ${ }^{3}$ and minimal improvement in functioning and symptomology. ${ }^{4}$ These disparities may be explained by structural barriers, such as homelessness and poverty, 
and systemic issues, as African American providers are scarce, and short-lived therapeutic relationships often result in distrust. ${ }^{6}$

Global migration, in particular the large diaspora of people of Chinese and South Asian (ie, Indian, Pakistani, Bangladeshi and Sri Lankan) descent, is increasingly shaping the demographic composition of high-income countries worldwide. ${ }^{7}$ However, there is a paucity of information on the effect of psychiatric hospitalisations on Asian ethnic groups living in Western countries, who may experience different socioeconomic conditions. South Asian and East Asian populations, in particular those of Chinese origin, have been shown to underutilise mental healthcare, ${ }^{8}$ even when experiencing severe and persistent mental illness. ${ }^{9}$ Studies also suggest that Chinese and South Asian patients present to hospital with greater mental illness severity, ${ }^{10}$ including higher rates of psychosis, aggressive behaviours and involuntary admissions, ${ }^{11}$ which may be consequences of lower outpatient service use and delayed help seeking. It is not known, however, whether these ethnic disparities remain at hospital discharge and how each of the Asian groups compare with the general population in their postdischarge outcomes.

In this population-based cohort study conducted in Ontario, the most populous province within Canada's single-payer universal healthcare system, where the Chinese and South Asian populations comprised 5\% and $7 \%$, respectively, in $2006,{ }^{12}$ we compared admission and discharge symptom severity measures and examined postdischarge outpatient service utilisation and outcomes, such as emergency department visits and psychiatric readmission or death, among all Chinese and South Asian psychiatric inpatients, as compared with inpatients of all other ethnicities.

\section{METHODS}

\section{Data sources}

We used data from the Ontario Mental Health Reporting System database (97\% record linkage rate), which contains information submitted by clinical staff on designated psychiatric beds across all hospitals in Ontario, ${ }^{13}$ and the Resident Assessment Instrument-Mental Health, a standardised minimum assessment clinical tool with demonstrated reliability and convergent validity, ${ }^{14}$ to capture information on several clinical rating scales and diagnoses according to the Diagnostic and Statistical Manual of Mental Disorders (DSM)-IV. We linked this dataset to the Canadian Institute for Health Information's Hospital Discharge Database (98\% record linkage rate), the Registered Persons Database, Ontario Health Insurance Plan physician billing database, the National Ambulatory Care Reporting System, and the Immigration, Refugees and Citizenship Canada-Permanent Resident database (86\% record linkage rate). Datasets were linked at the individual level using unique encoded identifiers and analysed at the Institute for Clinical Evaluative Sciences.

\section{Study population}

We examined all psychiatric inpatients who were discharged between 1 April 2006 and 31 March 2014, were Ontario residents, had valid health cards, were between the ages of 19 and 105 years, had voluntary or involuntary admissions and had data on psychiatric assessments at admission. We excluded hospitalisations with lengths of stay $\leq 3$ days (since diagnostic assessment data were not complete) or $>90$ days (as they reflect very complex patients who are not representative of the general hospitalised population). We also excluded patients with invalid diagnoses (eg, DSM-IV diagnoses listed as '7999' or starting with a letter) and other data inconsistencies. We limited the sample to the first admission for each patient within the study period in hierarchical order of severity using DSM-IV axis-1 discharge diagnosis: schizophrenia (295), bipolar disorder (296 except 296.2, 296.3, 296.9), depression (296.2, 296.3, 311) and all other diagnoses.

We used a validated surnames algorithm to classify patients as those of Chinese origin, South Asian origin or all other ethnicities (henceforth called the 'general population'). The algorithm ${ }^{15}$ was designed to optimise specificity (99.7\% for both ethnic groups) and positive predictive value (South Asian, 89.3\%; Chinese, 91.9\%) over sensitivity ( $50.4 \%$ and $80.2 \%$, respectively).

\section{Demographic/clinical characteristics}

We captured demographic characteristics, including age, sex, marital status, education, area-level income, immigration status and community size (greater/ smaller than 500000 residents). We also categorised patients as recent immigrants $(<10$ years in Canada), long-term immigrants $(\geq 10$ years $)$ or non-immigrants (eg, Canadian-born/immigrants prior to 1985) using the Immigration, Refugees and Citizenship Canada-Permanent Resident database. We further assessed whether patients were involuntarily hospitalised or detained for psychiatric assessment under the Ontario Mental Health Act and captured the reasons for admission (eg, threat to self, not mutually exclusive). Using information from clinical assessments at discharge, which reflect the final 72 hours of the hospitalisation, we captured primary diagnoses and the presence of a substance abuse diagnosis (DSM-IV 291.0-3/5/81/89/9, 292.0, 292.11-12/81-84/89/9, $303.00 / 90, \quad 304.00 / 10 / 20 / 30 / 40 / 50 / 60 / 80 / 90$, $305.00 / 10-90$ excluding 80 ), and calculated in-hospital length of stay.

\section{Mental illness severity}

Using the Resident Assessment Instrument-Mental Health, ${ }^{14}$ we examined, for both admission and discharge, several measures of psychiatric severity. The Aggressive Behaviour Scale and positive symptoms (hallucinations, command hallucinations, delusions and abnormal thought process or form) and negative symptoms (anhedonia, loss of interest, lack 
of motivation, and reduced interaction) scales (each ranging 0-12) and the Depression Rating Scale (ranging 0-14) have been described in detail elsewhere. ${ }^{11}$ Additionally, we examined Global Assessment of Functioning, a 100-point scale based on a clinician's rating of a patient's overall level of social, occupational and psychological functioning, with lower scores indicating poorer functioning.

\section{Healthcare utilisation and patient outcomes}

Using physician billings data, we examined postdischarge outpatient service utilisation, including visits to a psychiatrist, and mental health and addictions-related visits to family physicians or general practitioners using a validated algorithm. ${ }^{16}$

Our primary outcome was psychiatric hospital readmission, ascertained from hospital discharge data, or death by any cause within 30,180 and 365 days postdischarge. We also examined psychiatric emergency department visits and readmissions as secondary outcomes. Service utilisation and outcomes 30 days postdischarge were examined to assess the immediate consequences of hospitalisation, and later estimates were used to examine longer-term effects.

\section{Statistical analysis}

$\mathrm{X}^{2}$ tests were used to compare clinical and demographic characteristics, as well as illness severity measures at admission and at discharge in each of the Chinese and South Asian inpatient groups to the reference population. Student's t-test was used to examine age as a continuous variable.

For each of the $30 / 180 / 365$ day service utilisation and postdischarge outcomes, we used multivariable binary logistic regression models to examine the effects of each ethnic group, compared with the reference population, adjusted for age, sex, income, education, marital status, immigration status, community size, discharge diagnosis and diagnosis of substance abuse, as these variables differed between groups and were associated with postdischarge outcomes. ${ }^{17}$ To examine whether consistent results were obtained using time-toevent analysis, we performed Cox proportional hazards models for 1 year psychiatric hospital readmission or death adjusted for the aforementioned covariates, and produced Kaplan-Meier curves and adjusted survival curves using the corrected group prognosis method. ${ }^{18}$ Individuals were censored at loss of provincial health coverage (ascertained using the date of last healthcare contact) or 31 March 2014.

To further unpack the relationship between ethnicity and immigration status, as a sensitivity analysis, we conducted multivariable logistic regressions stratified by immigration status. We also stratified the analysis by sex and discharge diagnosis, and examined the impact of adding prior mental health hospitalisation in the past 5 years as a covariate in all logistic and Cox models.

All statistical analyses were conducted using SAS V.9.3.

\section{RESULTS}

\section{Demographic/clinical characteristics}

A total of 285379 inpatient hospital discharges in Ontario residents aged 19-105 years, who had a valid health card between 1 April 2006 and 31 March 2014, met our inclusion criteria. These correspond to 143854 unique individuals. After excluding 10 individuals for whom hospital episodes could not be created due to data inconsistencies (eg, non-unique admission dates), 3873 individuals with length of stay $>90$ days ( $1 \%$ of Chinese and $2 \%$ of South Asian inpatients), 645 individuals with length of stay $<3$ days (1\% excluded from each ethnic inpatient group) and 7193 individuals with missing/invalid discharge diagnoses, our study population comprised of 132133 unique patients: 2552 Chinese, 2439 South Asian and 127142 patients of all other ethnicities.

Chinese and South Asian inpatients were significantly younger than patients in the comparison group and were more likely to be married and to have completed postsecondary education (table 1 ). Compared with the reference group, Chinese patients were also more likely to be female. Asian patients were significantly more likely than general population patients to be diagnosed with schizophrenia and less likely to be diagnosed with substance abuse or depression; they also had shorter hospital stays by on average 2 days.

\section{Mental illness severity}

Although Asian patients presented with symptoms that were significantly worse at admission, their psychiatric severity measures were either reduced or on par with those of the comparison group at discharge (figure 1). Chinese and South Asian inpatients had greater improvements than other patients in measures of aggressive behaviour and positive symptoms, with the greatest absolute decreases in positive symptoms observed in Chinese patients (from $17 \%$ to $3.5 \%$, compared with $13.4 \%$ to $4.0 \%$ in South Asian patients and $9.2 \%$ to $2.1 \%$ in the reference group). At discharge, there were no significant differences between the Asian and the reference groups on the Aggressive Behaviour Scale $(\mathrm{P}>0.12)$. Both ethnic groups were also significantly less likely to maintain a poor score on the General Assessment of Functioning at discharge than the general population patients $(\mathrm{P}<0.004)$. By contrast, the relative change between admission and discharge in negative and depressive symptoms was equivalent across the three groups.

\section{Postdischarge service utilisation and patient outcomes}

Each of the Chinese and South Asian patients were similarly likely to seek outpatient mental healthcare postdischarge as the reference group (figure 2, supplementary table S1). However, examining service utilisation separately by physician specialties revealed that Chinese patients had greater adjusted odds of visiting a psychiatrist (OR 1.13, 95\% CI 1.02 to 1.25), as compared with the reference group, and South Asian patients had greater adjusted odds of visiting a general practitioner/family 
Table 1 Demographic and clinical characteristics of psychiatric inpatients by ethnic group

\begin{tabular}{|c|c|c|c|c|c|}
\hline & $\begin{array}{l}\text { Comparison group } \\
(n=127142)\end{array}$ & $\begin{array}{l}\text { Chinese } \\
(n=2552)\end{array}$ & P value* & $\begin{array}{l}\text { South Asian } \\
(\mathrm{n}=2439)\end{array}$ & P value ${ }^{*}$ \\
\hline Age at admission, mean (median) & $44.9(44)$ & $42.4(41)$ & $<0.001$ & $41.6(40)$ & $<0.001$ \\
\hline \multicolumn{6}{|l|}{ Age category } \\
\hline $19-44$ & $65222(51.3 \%)$ & 1492 (58.5\%) & $<0.001$ & $1486(60.9 \%)$ & $<0.001$ \\
\hline $45-64$ & $44646(35.1 \%)$ & $807(31.6 \%)$ & $<0.001$ & $716(29.4 \%)$ & $<0.001$ \\
\hline $65+$ & $17274(13.6 \%)$ & $253(9.9 \%)$ & $<0.001$ & $237(9.7 \%)$ & $<0.001$ \\
\hline \multicolumn{6}{|l|}{ Sex } \\
\hline Female & 63216 (49.7\%) & $1474(57.8 \%)$ & $<0.001$ & $1146(47.0 \%)$ & 0.007 \\
\hline Male & $63926(50.3 \%)$ & $1078(42.2 \%)$ & $<0.001$ & $1293(53.0 \%)$ & 0.007 \\
\hline \multicolumn{6}{|l|}{ Neighbourhood income quintile } \\
\hline Q1 (lowest) & 36178 (28.5\%) & 737 (28.9\%) & 0.64 & 722 (29.6\%) & 0.21 \\
\hline Q2 & $27049(21.3 \%)$ & $608(23.8 \%)$ & 0.002 & $604(24.8 \%)$ & $<0.001$ \\
\hline Q3 & 22651 (17.8\%) & 453 (17.8\%) & 0.93 & 505 (20.7\%) & $<0.001$ \\
\hline Q4 & $21286(16.7 \%)$ & $403(15.8 \%)$ & 0.20 & $372(15.3 \%)$ & 0.05 \\
\hline Q5 (highest) & $19183(15.1 \%)$ & $334(13.1 \%)$ & 0.005 & $232(9.5 \%)$ & $<0.001$ \\
\hline Missing & $795(0.6 \%)$ & $17(0.7 \%)$ & 0.80 & $\leq 5(0.2 \%)$ & 0.004 \\
\hline \multicolumn{6}{|l|}{ Education } \\
\hline Less than high school & 30095 (23.7\%) & 372 (14.6\%) & $<0.001$ & 477 (19.6\%) & $<0.001$ \\
\hline High school graduate & $33398(26.3 \%)$ & $454(17.8 \%)$ & $<0.001$ & $598(24.5 \%)$ & 0.05 \\
\hline Any postsecondary & $50420(39.7 \%)$ & $1315(51.5 \%)$ & $<0.001$ & $1088(44.6 \%)$ & $<0.001$ \\
\hline Unknown & $13229(10.4 \%)$ & 411 (16.1\%) & $<0.001$ & 276 (11.3\%) & 0.15 \\
\hline \multicolumn{6}{|l|}{ Marital status } \\
\hline Single & $57302(45.1 \%)$ & 1266 (49.6\%) & $<0.001$ & 1010 (41.4\%) & $<0.001$ \\
\hline Married common law & $41746(32.8 \%)$ & $951(37.3 \%)$ & $<0.001$ & $1089(44.6 \%)$ & $<0.001$ \\
\hline Widowed, separated, divorced & $28094(22.1 \%)$ & $335(13.1 \%)$ & $<0.001$ & $340(13.9 \%)$ & $<0.001$ \\
\hline \multicolumn{6}{|l|}{ Immigration status } \\
\hline Immigrant $<10$ years in Canada & $3556(2.8 \%)$ & $486(19.0 \%)$ & $<0.001$ & $396(16.2 \%)$ & $<0.001$ \\
\hline Immigrant $10+$ years in Canada & $6804(5.4 \%)$ & $657(25.7 \%)$ & $<0.001$ & $764(31.3 \%)$ & $<0.001$ \\
\hline Non-immigrant & $116782(91.9 \%)$ & $1409(55.2 \%)$ & $<0.001$ & $1279(52.4 \%)$ & $<0.001$ \\
\hline \multicolumn{6}{|l|}{ Community size } \\
\hline$<500000$ & $66350(52.2 \%)$ & $307(12.0 \%)$ & $<0.001$ & $224(9.2 \%)$ & $<0.001$ \\
\hline$\geq 500000$ & $60792(47.8 \%)$ & $2245(88.0 \%)$ & $<0.001$ & $2215(90.8 \%)$ & $<0.001$ \\
\hline \multicolumn{6}{|l|}{ Admission status } \\
\hline Voluntary & $68793(54.1 \%)$ & $836(32.8 \%)$ & $<0.001$ & $987(40.5 \%)$ & $<0.001$ \\
\hline Involuntary & $58349(45.9 \%)$ & $1716(67.2 \%)$ & $<0.001$ & $1452(59.5 \%)$ & $<0.001$ \\
\hline \multicolumn{6}{|c|}{ Reason for admission (not mutually exclusive) } \\
\hline Threat to self & $61680(48.5 \%)$ & $1274(49.9 \%)$ & 0.16 & $1107(45.4 \%)$ & 0.002 \\
\hline Threat to others & $21053(16.6 \%)$ & 620 (24.3\%) & $<0.001$ & 544 (22.3\%) & $<0.001$ \\
\hline Inability to care for self & $45267(35.6 \%)$ & $1106(43.3 \%)$ & $<0.001$ & $1055(43.3 \%)$ & $<0.001$ \\
\hline Problem with addiction & $35262(27.7 \%)$ & $178(7.0 \%)$ & $<0.001$ & $447(18.3 \%)$ & $<0.001$ \\
\hline Justice system & 4946 (3.9\%) & 95 (3.7\%) & 0.67 & $103(4.2 \%)$ & 0.40 \\
\hline \multicolumn{6}{|c|}{ Primary diagnosis† (hierarchical, mutually exclusive) } \\
\hline Schizophrenia & $25454(20.0 \%)$ & $898(35.2 \%)$ & $<0.001$ & 739 (30.3\%) & $<0.001$ \\
\hline Bipolar disorder & $18773(14.8 \%)$ & $292(11.4 \%)$ & $<0.001$ & $341(14.0 \%)$ & 0.28 \\
\hline Depression & 35547 (28.0\%) & 655 (25.7\%) & 0.01 & 633 (26.0\%) & 0.03 \\
\hline Other & $47368(37.3 \%)$ & 707 (27.7\%) & $<0.001$ & 726 (29.8\%) & $<0.001$ \\
\hline
\end{tabular}


Table 1 Continued

\begin{tabular}{|c|c|c|c|c|c|}
\hline & $\begin{array}{l}\text { Comparison group } \\
(n=127142)\end{array}$ & $\begin{array}{l}\text { Chinese } \\
(n=2552)\end{array}$ & P value* & $\begin{array}{l}\text { South Asian } \\
(n=2439)\end{array}$ & P value* \\
\hline Substance abuse diagnosis† & $31978(25.2 \%)$ & $157(6.2 \%)$ & $<0.001$ & $355(14.6 \%)$ & $<0.001$ \\
\hline Mean $\pm S D$ & $20.20 \pm 16.68$ & $18.93 \pm 15.69$ & $<0.001$ & $17.80 \pm 14.58$ & $<0.001$ \\
\hline Median (IQR) & $15(8-27)$ & $14(8-24)$ & $<0.001$ & $13(8-23)$ & $<0.001$ \\
\hline
\end{tabular}

${ }^{*} \chi^{2}$ test comparing each ethnic group with the general population (Student's t-test was used to examine age as a continuous variable).

†Diagnosis at discharge.

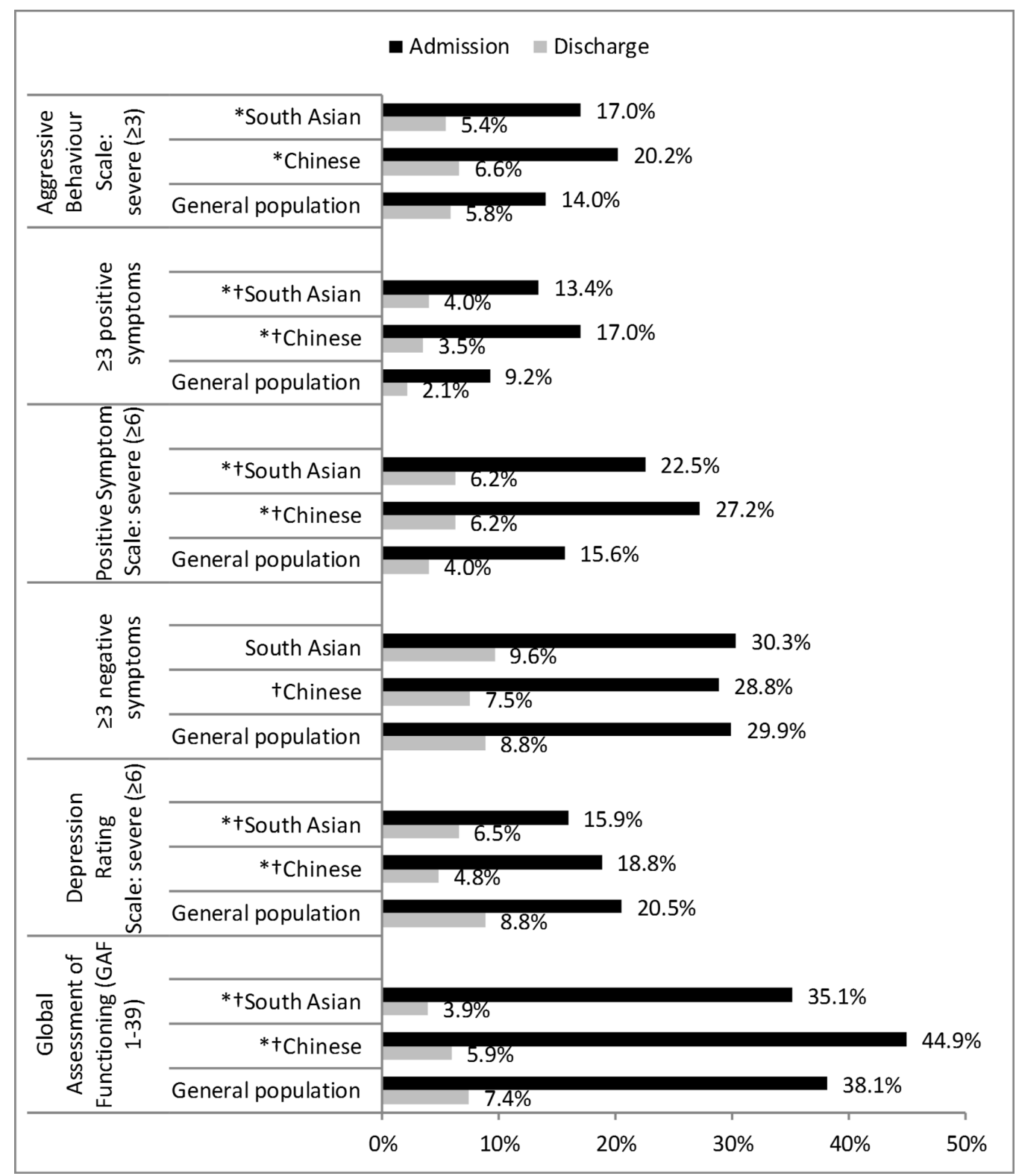

Figure 1 Resident Assessment Instrument-Mental Health severity measures at admission and discharge, by ethnic group. *Statistically significant difference $\left(\mathrm{P}<0.05, \chi^{2}\right.$ test) compared with the general population patients at admission. †Statistically significant difference $\left(\mathrm{P}<0.05, \chi^{2}\right.$ test) compared with the general population patients at discharge. Percentages are out of each ethnic group (general population $n=127561$, Chinese $n=2556$, South Asian $n=2443$ ). 
Outcome

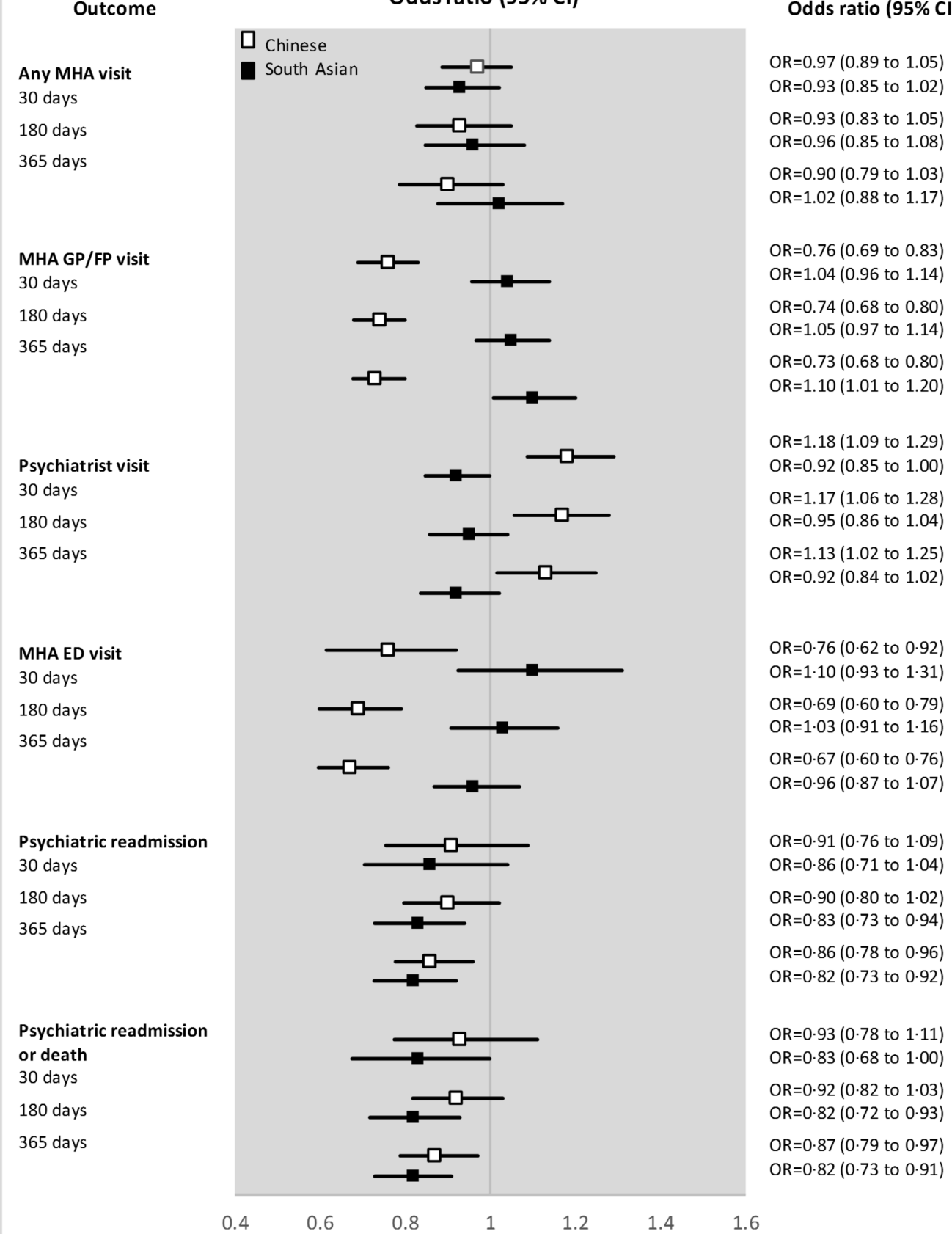

Figure 2 Adjusted OR of service utilisation and patient outcomes 30/180/365 days postdischarge, in Chinese and South Asian psychiatric patients, compared with the general population patients. Logistic models adjusted for age, sex, income, education, marital status, immigration status, community size, discharge diagnosis and diagnosis of substance abuse; numbers of events for each ethnic group are provided in supplementary table S1. ED, emergency department; GP/FP, general practitioner/family physician; MHA, mental health and addictions.

physician for mental health services 1-year postdischarge (OR $1.10,95 \%$ CI 1.01 to 1.20 ), compared with the reference group. The Asian groups also showed better postdischarge outcomes as compared to the reference population (figure 2), with Chinese patients having 13\% (OR $0.87,95 \%$ CI 0.79 to 0.97 ) and South Asians patients having $18 \%$ (OR $0.82,95 \%$ CI 0.73 to 0.91 ) lower adjusted odds of psychiatric readmission or death at 1 year of follow-up. Chinese patients had also lower adjusted odds of visiting the emergency department for mental health reasons 1 year following discharge (OR $0.67,95 \% \mathrm{CI}$ 0.60 to 0.76$)$ and South Asian patients had similar odds $(\mathrm{P}=0.40)$, compared with general population patients.

The Kaplan-Meier curve and unadjusted Cox proportional hazards models (supplementary figure S1) showed similar or lower hazards of psychiatric readmission or death among Chinese and South Asian patients. After controlling for sociodemographic and clinical factors, we 


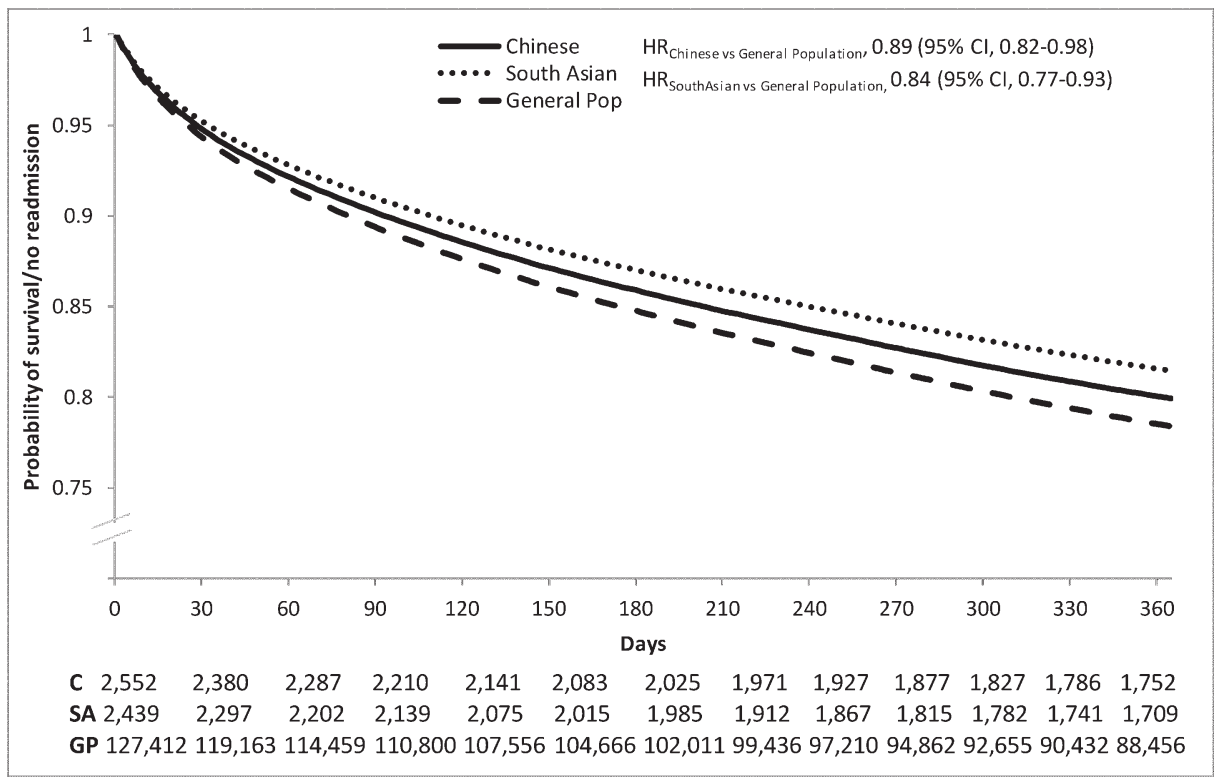

Figure 3 Adjusted survival plots of time between discharge (time 0) and psychiatric readmission or death, by ethnic group, and HRs comparing each group with the general population. Curve adjusted for age, sex, income, education, marital status, immigration status, community size, discharge diagnosis and diagnosis of substance abuse using the corrected group prognosis method; individuals were censored at loss of provincial health coverage or 31 March 2014. HRs were generated using a Cox proportional hazards model adjusted for the aforementioned demographic and clinical characteristics. C, Chinese; GP, general practitioner; Gen pop, general population; SA, South Asian.

found significantly reduced hazards of psychiatric readmission or all-cause mortality among Chinese (HR 0.89, $\mathrm{P}=0.01$ ) and South Asian (HR 0.84, $\mathrm{P}<0.001$ ) patients compared with the reference group (figure 3 ).

Sensitivity analyses revealed that the odds of psychiatric readmission or death 365 days postdischarge were similar between males and females and across diagnoses and immigrant groups (supplementary table S2). Lastly, our findings were consistent even after additional adjustment for prior mental health hospitalisation in the past 5 years.

\section{DISCUSSION}

Patients of Chinese and South Asian origin experienced greater absolute improvements during their psychiatric hospitalisations compared with other patients, as evidenced by improved psychiatric symptomology and general functioning at discharge, despite presenting with greater severity at admission. They had also shorter lengths of hospital stay and better postdischarge outcomes, including significantly reduced likelihood of emergency department visits and psychiatric readmissions or death, even after controlling for sociodemographic and clinical characteristics. Once discharged, they were similarly or more likely to seek mental healthcare in outpatient settings as the comparison group.

This study has several strengths. First, to our knowledge, this is the largest population-based study to examine ethnic differences in postdischarge outcomes using administrative healthcare databases. Second, Canada has the highest proportion of immigrants of any G8 country ${ }^{19}$ and has one of the largest Asian populations outside of Asia, making it an ideal setting to study cross-cultural differences. Third, the availability of many covariates including diagnostic and sociodemographic factors allowed us to examine the independent effect of ethnicity. We were also able to ascertain that the results were not attributable to differences by sex, diagnosis or immigration status, and control for potential financial barriers as all study participants were covered under Canada's single-payer healthcare system. Lastly, we were able to examine Chinese and South Asian patients separately, unlike other studies that combined these and other ethnic groups. However, some limitations are worth noting. First, the surnames algorithm for ascertaining ethnicity was highly specific but had low sensitivity. As a result, some Chinese and South Asian inpatients may have been misclassified as belonging to the general population, which may have underestimated the true effect. Second, the reference group is very heterogeneous, as it encompassed all other ethnicities, including black African and Caribbean inpatients, who are known to have worse outcomes than other groups, which may have drawn the results in either direction. Lastly, although there is heterogeneity in the South Asian group, this study examined Chinese and South Asian patients separately, revealing important differences in service utilisation and outcomes. Future research is needed to ascertain the full trajectory of postdischarge care in these populations by including information on contacts with community mental health services and other supports, such as family networks, which were not available in our administrative data holdings. 
European and American studies found that black patients present to psychiatric hospitals with more severe symptoms than comparison groups ${ }^{5}$ and that they do not experience much improvement at the time of or following discharge. ${ }^{34}$ By contrast, our findings among Chinese and South Asian patients illustrate significant improvements through hospitalisation and sustained gains postdischarge that are on par with or exceed those of other patients. Although comparable results have been suggested, the few available studies have produced mixed results and have often been limited by small samples, cross-sectional designs, subjective outcome measures and considerable heterogeneity within ethnic categories. ${ }^{10} 20$ While the evidence on length of stay is mixed, ${ }^{22}{ }^{23}$ one study found no difference between Asian and white patients with regard to psychiatric symptomology closely following discharge, ${ }^{10}$ and another showed that Asian patients experienced the greatest decline in psychiatric severity scores between admission and discharge compared with Caucasians and Pacific Islanders. ${ }^{21}$ Another study found that although Asian patients were admitted with similar levels of functional impairment as the general population, they scored higher on the Global Assessment of Functioning scale at discharge. ${ }^{22}$ Since South Asian and other ethnic groups living with mental illness in Canada were shown to have unmet mental healthcare needs, ${ }^{24}$ lower rates of acute care visits postdischarge may suggest continued delay of care or additional issues around access, but the increased utilisation of outpatient services we observed illustrates a positive service engagement trajectory following discharge. Similarities between Asian and white psychiatric patients with regard to outpatient follow-up postdischarge have been previously suggested, ${ }^{25}$ with higher rates of general practitioner visits in South Asians, ${ }^{10}$ and greater psychiatrist utilisation in Chinese patients with severe mental illness. ${ }^{9}$ Although a greater proportion of Chinese patients, as compared with South Asian and general population patients, were diagnosed with schizophrenia, our study illustrates that increased psychiatrist use postdischarge among Chinese patients does not indicate greater illness severity, given that acute presentations were lower in this group. Previous small studies similarly observed comparable or lower rates of hospital readmission 1 year postdischarge in Asian, as compared with white and Afro-Caribbean patients. ${ }^{20} 21$ We therefore highlight that receiving intensive inpatient treatment leads to increased outpatient service contact and positive outcomes among Asian patients.

Our findings may be explained by individual, cultural and health system characteristics. With the most common point of access for black African patients being crisis services and police apprehension, patients' distrust may be compounded, resulting in early treatment termination, reduced follow-up contact and poor outcomes. ${ }^{5}$ By contrast, Asian psychiatric patients in Canada were shown to be more likely than black individuals to use the emergency department as the first point of contact rather than the justice system. ${ }^{26}$ Poor outcomes among black patients in other countries may also be attributed to structural and systemic issues, including limited coverage by medical insurance and living in poverty. ${ }^{6}$ By contrast, the Chinese and South Asian patients in this study were covered by Canada's universal healthcare system, ${ }^{27}$ were more likely to have completed higher education and had a similar income distribution as the comparison group. However, although individuals of Chinese and South Asian origin comprised $5 \%$ and $7 \%$ of the Ontario population, respectively, ${ }^{12}$ they each represented $2 \%$ of the inpatient population in our study, which may reflect access issues or delayed care seeking. ${ }^{11}$

It may also be that while some cultural factors impede mental health care seeking prior to hospitalisation, others may positively impact treatment engagement postdischarge. Previous studies have posited that the greater illness severity among Asian patients at admission is a result of cultural stigma and reluctance to seek help, ${ }^{28}$ yet cultural-specific factors that might prevent readmission such as extended family networks and lower prevalence of substance abuse have also been described. ${ }^{29} 30$ Reduced stigmatisation following discharge, coupled with strong family support and conscientiousness, may also enable and empower patients to maintain outpatient services. Furthermore, a recent report by the Mental Health Commission of Canada has highlighted the cultural incompatibility of existing services as the most pertinent access barrier for racialised populations and called for the development of culturally appropriate supports. ${ }^{31}$ Hospital-based providers in Canada, as compared with the USA, have been shown to practice cultural competency, ${ }^{32} 33$ which has been tied to positive patient outcomes. ${ }^{3435}$ However, while one pilot project in Southern Ontario illustrated that a 5-week educational programme was effective in increasing Public Health Nurses' cultural knowledge at follow-up, ${ }^{33}$ the extent to which cultural competency extends to psychiatric settings is not known. Assigning individuals to ethnically focused inpatient units has also been shown to increase the likelihood of patients accepting outpatient referrals and decrease the likelihood of readmissions. ${ }^{35} 36$ The combination of reduced acute care encounters and increased outpatient service utilisation postdischarge suggests that there are opportunities to reduce prolonged suffering. Future studies are needed to investigate whether earlier intervention targeted towards Asian communities may be effective in reducing stigma and empowering patients and families to seek help early.

In conclusion, despite being admitted with more severe symptoms and behaviours, patients of Chinese and South Asian origin fared as well as or better than the general population patients at discharge and postdischarge. This study has important implications for high-income countries that have large Chinese and South Asian ethnic populations and for physicians who provide care in multiethnic practices, as both cultural and structural factors may be contributing to the observed findings. Further investigation into the patient-level and system-level factors 
which yield positive outcomes and a positive trajectory of service engagement following hospitalisations may help eliminate ethnic disparities in mental healthcare.

Acknowledgements We thank the Ontario Mental Health Reporting System, the Immigration, Refugees and Citizenship Canada, and the Canadian Institute for Health Information for their data. Parts of this material are based on data compiled and provided by the Canadian Institute for Health Information. However, the analyses, conclusions, opinions and statements expressed herein are those of the authors and are independent from the Ontario Mental Health Reporting System, the Immigration, Refugees and Citizenship Canada, and the Canadian Institute for Health Information.

Contributors $\mathrm{MC}$ was the principal investigator and $\mathrm{MC}$ and $\mathrm{ML}$ were responsible for the conception and design of this study. LF and AN analysed the data and all authors (MC, ML, LF, AN, EG, JZ, PK) interpreted the data. MC and EG drafted the manuscript and all authors (MC, EG, ML, LF, AN, JZ, PK) critically revised it and approved the final manuscript. All authors (MC, EG, JZ, ML, LF, AN, PK) had full access to the study results and assume full responsibility for the accuracy and completeness of the ideas presented. $\mathrm{MC}$ is the guarantor and takes responsibility for the integrity of the data and the accuracy of the data analysis.

Funding This study was supported by the Institute for Clinical Evaluative Sciences (ICES, Ontario, Canada), which is funded by an annual grant from the Ontario Ministry of Health and Long- Term Care (MOHLTC). It was also supported by the Mental Health and Addictions Scorecard and Evaluation Framework grant from the MOHLTC (grant number 04601A14-19). However, the opinions, results and conclusions reported in this paper are those of the authors and are independent from the funding sources. No endorsement by ICES or the Ontario MOHLTC is intended or should be inferred. The funders had no role in the design, analysis, interpretation or publication of this study.

Competing interests None declared.

Ethics approval Ethics approval for this study was obtained from the Research Ethics Board at Sunnybrook Health Sciences Centre.

Provenance and peer review Not commissioned; externally peer reviewed.

Data sharing statement № additional data are available.

Open Access This is an Open Access article distributed in accordance with the Creative Commons Attribution Non Commercial (CC BY-NC 4.0) license, which permits others to distribute, remix, adapt, build upon this work non-commercially, and license their derivative works on different terms, provided the original work is properly cited and the use is non-commercial. See: http://creativecommons.org/ licenses/by-nc/4.0/

(c) Article author(s) (or their employer(s) unless otherwise stated in the text of the article) 2018. All rights reserved. No commercial use is permitted unless otherwise expressly granted.

\section{REFERENCES}

1. Thornicroft G, Tansella M. Components of a modern mental health service: a pragmatic balance of community and hospital care: overview of systematic evidence. Br J Psychiatry 2004;185:283-90.

2. Weiden PJ, Kozma C, Grogg A, et al. Partial compliance and risk of rehospitalization among California Medicaid patients with schizophrenia. Psychiatr Serv 2004;55:886-91.

3. Carson NJ, Vesper A, Chen CN, et al. Quality of follow-up after hospitalization for mental illness among patients from racial-ethnic minority groups. Psychiatr Serv 2014;65:888-96.

4. Eack SM, Newhill CE. Racial Disparities in Mental Health Outcomes After Psychiatric Hospital Discharge Among Individuals With Severe Mental Illness. Soc Work Res 2012;36:41-52.

5. Bhui K, Bhugra D. Mental illness in Black and Asian ethnic minorities: pathways to care and outcomes. Advances in Psychiatric Treatment 2002;8:26-33.

6. Satcher D. Mental health: Culture, race, and ethnicity -A supplement to mental health: A report of the surgeon general: Chapter 3 Mental Health Care for African Americans. Rockville MD: Substance Abuse and Mental Health Services Administration, 2001:51-76.

7. Department of Economic and Social Affairs. The number of international migrants worldwide reaches 232 million. Population Division, United Nations. 2013 http://www.un.org/en/development/ desa/news/population/number-of-international-migrants-rises.html (accessed May 2016).
8. Tiwari SK, Wang J. Ethnic differences in mental health service use among White, Chinese, South Asian and South East Asian populations living in Canada. Soc Psychiatry Psychiatr Epidemiol 2008:43:866-71.

9. Chen AW, Kazanjian A, Wong $\mathrm{H}$, et al. Mental health service use by Chinese immigrants with severe and persistent mental illness. Can J Psychiatry 2010;55:35-42.

10. Commander MJ, Cochrane R, Sashidharan SP, et al. Mental health care for Asian, black and white patients with non-affective psychoses: pathways to the psychiatric hospital, in-patient and after-care. Soc Psychiatry Psychiatr Epidemiol 1999:34:484-91.

11. Chiu M, Lebenbaum M, Newman AM, et al. Ethnic Differences in Mental Illness Severity: A Population-Based Study of Chinese and South Asian Patients in Ontario, Canada. J Clin Psychiatry 2016;77:e1108-16.

12. Statistics Canada. Population by selected ethnic origins, by province and territory (2006 Census)(Ontario). 2006http://www.statcan.gc.ca/ tables-tableaux/sum-som/l01/cst01/demo26g-eng.htm (accessed Sep 2017).

13. Canadian Institute for Health Information. Exploring Hospital Mental Health Service Use in Ontario, 2007-2008. https://secure.cihi.ca/ free products/OMHRS aib en.pdf

14. Hirdes JP, Smith TF, Rabinowitz T, et al. The Resident Assessment Instrument-Mental Health (RAI-MH): inter-rater reliability and convergent validity. J Behav Health Serv Res 2002;29:419-32.

15. Shah BR, Chiu M, Amin S, et al. Surname lists to identify South Asian and Chinese ethnicity from secondary data in Ontario, Canada: a validation study. BMC Med Res Methodol 2010;10:42.

16. Steele LS, Glazier RH, Lin E, et al. Using administrative data to measure ambulatory mental health service provision in primary care. Med Care 2004:42:960-5.

17. Donisi V, Tedeschi F, Wahlbeck K, et al. Pre-discharge factors predicting readmissions of psychiatric patients: a systematic review of the literature. BMC Psychiatry 2016;16:449.

18. Ghali WA, Quan H, Brant R, et al. Comparison of 2 methods for calculating adjusted survival curves from proportional hazards models. JAMA 2001;286:1494-7.

19. Statistics Canada. Immigration and Ethnocultural Diversity in Canada: National Household Survey, 2011 Statistics Canada website 2013. 2013. http://www12.statcan.gc.ca/nhs-enm/2011/as-sa/99010-x/99-010-x2011001-eng.pdf (accessed Apr 2016).

20. Bhui K, Stansfeld S, Hull S, et al. Ethnic variations in pathways to and use of specialist mental health services in the UK. Systematic review. Br J Psychiatry 2003;182:105-16.

21. Anders RL, Olson T, Bader J. Assessment of acutely mentally ill patient outcomes in an ethnically diverse population. Int J Psychiatr Nurs Res 2006:11:1331-47.

22. Kim JE, Saw A, Zane NW, et al. Patterns of Utilization and Outcomes of Inpatient Psychiatric Treatment in Asian Americans. Asian Am J Psychol 2014;5:35-43.

23. Shin JK. Inpatient stays of Asian patients with psychiatric diagnoses in New York City. Issues Ment Health Nurs 2009;30:112-21.

24. Gadalla TM. Ethnicity and seeking treatment for depression: A Canadian National Study. Can Ethn Stud 2010;41:233-45.

25. Anderson KK, Fuhrer R, Schmitz N, et al. Determinants of negative pathways to care and their impact on service disengagement in first-episode psychosis. Soc Psychiatry Psychiatr Epidemiol 2013;48:125-36.

26. Archie S, Akhtar-Danesh N, Norman R, et al. Ethnic diversity and pathways to care for a first episode of psychosis in Ontario. Schizophr Bull 2010;36:688-701.

27. Lasser KE, Himmelstein DU, Woolhandler S. Access to care, health status, and health disparities in the United States and Canada: results of a cross-national population-based survey. Am J Public Health 2006:96:1300-7.

28. Thomson MS, Chaze F, George U, et al. Improving Immigrant Populations' Access to Mental Health Services in Canada: A Review of Barriers and Recommendations. J Immigr Minor Health 2015;17:1895-905

29. Birchwood M, Cochrane R, Macmillan F, et al. The influence of ethnicity and family structure on relapse in first-episode schizophrenia. A comparison of Asian, Afro-Caribbean, and white patients. Br J Psychiatry 1992;161:783-90.

30. Satcher D. Mental health: Culture, race, and ethnicity-A supplement to mental health: A report of the surgeon general: Chapter 5 Mental Health Care for Asian Americans and Pacific Islanders. Rockville MD: Substance Abuse and Mental Health Services Administration (US), 2001. 
31. Mental Health Commission of Canada. The Case for Diversity: Building the Case to Improve Mental Health Services for Immigrant, Refugee, Ethno-cultural and Racialized Populations. Ottawa, ON: Mental Health Commission Of Canada, 2016.

32. Schim SM, Doorenbos AZ, Borse NN. Cultural competence among Ontario and Michigan healthcare providers. J Nurs Scholarsh 2005;37:354-60.

33. Brathwaite AC, Majumdar B. Evaluation of a cultural competence educational programme. J Adv Nurs 2006;53:470-9.
34. Smith TB, Constantine MG, Dunn TW, et al. Multicultural education in the mental health Professions: A meta-analytic review. J Couns Psychol 2006;53:132-45.

35. Meyer OL, Zane N. The influence of race and ethnicity in clients' experiences of mental health treatment. J Community Psychol 2013;41:884-901.

36. Mathews CA, Glidden D, Murray S, et al. The effect on treatment outcomes of assigning patients to ethnically focused inpatient psychiatric units. Psychiatr Serv 2002;53:830-5. 